\title{
Análisis aerodinámico regional mediante cfd de un semirremolque cisterna para transporte de cemento
}

\author{
Regional aerodynamic analysis by the means cfd of a tanker trailer to \\ transport cement \\ Abel Remache Coyago ${ }^{1}$, Luis Tipanluisa Sarchi ${ }^{2}$, Juan Salvador Jiménez ${ }^{3}$ y \\ WASHINTON ERAZO LAVERDE ${ }^{4}$
}

\section{RESUMEN}

Se realizó un análisis del flujo de aire de un camión transportador de cemento en la región Sierra - Riobamba y la región Costa - Guayaquil, con la finalidad de investigar su intervención e influencia en los siguientes coeficientes y factores: Drag o arrastre $\left(C_{D}\right)$, Lift o elevación $\left(\mathrm{C}_{\mathrm{T}}\right)$, Resistencia aerodinámica $\left(\mathrm{P}_{2}\right)$ y Potencia para vencer la resistencia al aire (R). Este análisis se efectuó mediante el uso de la mecánica de Fluidos Computacional (CFD). Para esto, se desarrolló un modelo geométrico y 3D del semirremolque y del tracto camión. Los datos del fluido fueron características promedio del aire y microclimas. Se obtuvo un $C_{D}$ bajo en la zona costera, un $C_{L}$ similar en cada caso, una $P_{a}$ superior en la zona costera, y una $\mathrm{R}$ superior en Guayaquil, siendo estos valores lógicos en un buen diseño aerodinámico. Se concluye que $\mathrm{R}$ es mayor en la zona costera, pero la concentración de aire y la presión atmosférica pueden influir en la combustión y por ende en la adición de potencia, viéndose compensada de alguna manera esta relación. $\mathrm{C}_{\mathrm{D}}$ es inferior en la zona costera, con similar arrastre de forma y fricción; la densidad ayuda a mejorar notablemente este coeficiente.

Palabras clave: coeficiente de sustentación; coeficiente de arrastre; aerodinámica de camiones; CFD; resistencia del aire.

\footnotetext{
ABSTRACT using computational fluid mechanics (CFD).

Universidad Central del Ecuador. Quito, Ecuador.

2 Escuela Politécnica Nacional. Quito, Ecuador.

3 Universidad Internacional SEK. Quito, Ecuador.

4 Universidad de las Fuerzas Armadas. Latacunga, Ecuador.
}

An airflow analysis of a hauler of cement in the mountain region - Guayaquil was performed, for the purpose of investigation into its role and influence in the following coefficients and factors: Drag or drag (CD) lift or elevation (CL), aerodynamic resistance $(\mathrm{Pa})$, and power to overcome air resistance $(\mathrm{R})$. This analysis was performed 
For this, a 3D geometric model and the semi-trailer and truck tract developed. The data were averaged fluid characteristics of air and microclimates. A lower CD in the coastal zone, a similar CL in each case, a higher $\mathrm{Pa}$ in the coastal area, and a higher $\mathrm{R}$ Guayaquil, and these logical values in a good aerodynamic design was obtained. It is concluded that $\mathrm{R}$ is higher in the coastal zone, but the concentration of air and atmospheric pressure can influence the combustion and therefore the addition of power, looking compensated somehow this relationship. CD is lower in the coastal zone, having similar towed shape and frictio, density helps to significantly improve this ratio.

Keywords: lift coefficient; drag coefficient; aerodynamic truck; CFD; air resistance.

\section{ICHIKLLACHAW}

Simintu apaq kamiyunpita wayra yarquqtam rikapashqa kashqa, tsay kashqa hirka markakunachaw - Riobamba niykur achachak markakunachaw - Guayaquilchawmi; tsay rurakashqa coeficienteswan facturkuna imanawpis kay qatiqkunawan tanqanakuyanqanta rikaanapaq: Drag aw qarachana (CD), Lift aw umayaanan (CL), Tsarakuq wayra dinámika $(\mathrm{Pa})$, wayrata llallinapaq kallpata $(\mathrm{R})$. Kay rikapakuy rurakashqa mikanika Fluidusta Kumputasiyunalta iñishirmi (CFD). Tsaypaq rurakashqa huk mudilu hiyumitriku huk 3D pullanrremulkipa niykur kamiyunpa sutaqnitawan. Tsay yarqamuq wayrapawan microclimankuna pullantsashqa. Yunka kaqchaw yarkushqa CD, CL tsaynawlla llapanchaw, huk Pa llallip achachap markachaw, niykur huk R llallip Guayaquilchaw, kaynawmi allí tsanintsay huk alli diseño aerodinámicuchaw. Ushpanaq kay $\mathrm{R}$ yunkachawmi atska, itsa atska wayrawan patsa nitiqwan tanqan rawrayninta, tsay raykur hina adición de putinsiya yapanan, yanapanaqkuyan kay kaqkuna. CD yunkachaw ichikllam, arrastriwan kupayqa tsaynawllam, densidadmi yanapakun kay coeficiente qarachaqwan.

Pushaq shimikuna: kuwihisiyinti saraqkuna; kuwihisiyinti rutataqkuna; kamiyunkunapa wayradinamikan; CFD; wayra tsaraanan.

\section{INTRODUCCIÓN}

La Simulación Aerodinámica mediante técnicas de CFD de un semirremolque cisterna para el transporte de cemento, tiene como objetivo intervenir en la optimización del consumo de combustible, que es destinado a los vehículos que realizan el transporte de productos en grandes cantidades por vía terrestre, con el afán de colaborar en investigaciones futuras de consumo de combustibles y técnicas de simulación computacional para camiones. Además, internamente se colabora con los lineamientos del Plan Nacional de Buen Vivir - Ecuador (SENPLADES, 2013).

Los puntos fundamentales tomados en cuenta para el análisis son los siguientes: 
La resistencia aerodinámica del vehículo, siendo esta la fuerza que se opone al avance a través del aire, viene determinada por:

$$
\mathrm{Pa}=\frac{\rho}{2} \mathrm{C}_{\mathrm{x}} \mathrm{A}_{\mathrm{F}} \mathrm{v}_{\mathrm{r}}^{2}
$$

Donde:

$\rho=$ Densidad del Aire, $C_{\mathrm{x}}=$ Coeficiente de resistencia aerodinámica, $A_{\mathrm{F}}=$ Área Frontal del vehículo proyectada en la dirección del movimiento y $\mathrm{v}_{\mathrm{r}}=$ Velocidad.

De forma general, el coeficiente $C_{x}$ se obtiene en túneles de viento con prototipos a escala o vehículos a escala real. Las escalas comunes en Europa son 1/4 o 1/5 para vehículos convencionales, y en vehículos comerciales se recomienda la escala $1 / 2,5$ (Yung, 2001).

La fuerza de arrastre, que se contrarresta por medio de la fuerza de propulsión en la dirección opuesta, da como resultado el incremento o mantenimiento de la velocidad del vehículo. Esta fuerza queda representada por:

$$
\mathrm{F}_{\mathrm{D}}=\mathrm{C}_{\mathrm{D}} \mathrm{X} \frac{1}{2} \rho \mathrm{v}^{2} \mathrm{~A}
$$

Donde:

$\mathrm{F}_{\mathrm{D}}=$ Arrastre $(\mathrm{N}), \rho=$ Densidad del Fluido $\left(\mathrm{kg} / \mathrm{m}^{3}\right), \mathrm{v}=$ Velocidad del fluido $(\mathrm{m} / \mathrm{s})$, $\mathrm{A}=$ Superficie frontal del cuerpo perpendicular al movimiento del fluido $\left(\mathrm{m}^{2}\right)$ y $C_{D}=$ Coeficiente de Arrastre (adimensional) (Mott, 2006).

La sustentación es el componente aerodinámico perpendicular a la dirección en la que se traslada un cuerpo, dirigido verticalmente hacia arriba o abajo. El coeficiente de sustentación es un valor que permite comparar su incidencia-desempeño en cuerpos con diferentes formas y tamaños. Un coeficiente de sustentación positivo representa agarre o apoyo, mientras que uno negativo representa carga aerodinámica. Esta fuerza queda representada por:

$$
\mathrm{F}_{\mathrm{L}}=\mathrm{C}_{\mathrm{L}} \mathrm{X} \frac{1}{2} \rho \mathrm{v}^{2} \mathrm{~A}
$$

Donde:

$\mathrm{F}_{\mathrm{D}}=$ Sustentación $(\mathrm{N}), \rho=$ Densidad del Fluido $\left(\mathrm{kg} / \mathrm{m}^{3}\right), \mathrm{v}=$ Velocidad del Fluido $(\mathrm{m} / \mathrm{s}), \mathrm{A}=$ Superficie frontal del Cuerpo perpendicular al movimiento del fluido $\left(\mathrm{m}^{2}\right)$, $\mathrm{C}_{\mathrm{L}}=$ Coeficiente de Sustentación (adimensional) (Mc. Beath, 2005).

Se toma en cuenta para el análisis ciertas leyes de la Aerodinámica tales como:

El efecto Coanda, que es la tendencia de una corriente de aire o fluido a adherirse a las paredes del vehículo, la comprensión de este efecto permite poseer un criterio técnico 
que ayuda de manera significativa al desarrollo de soluciones y conclusiones en el análisis que se realiza sobre todo en camiones (Coanda, 2004).

Estela, que se define como la línea de corriente que coincide en forma paralela con la pared. La región debajo de la línea de corriente que parte del punto de separación se llama estela (Streeter y Wylie, 1980). La aerodinámica en camiones puede ofrecer variados resultados y formas en la estela generada dependiendo del diseño del camión transportador.

Capa Limite, a partir del inicio del movimiento de un fluido de muy baja viscosidad, la rotación del flujo es nula. Dado que el fluido en las fronteras sólidas tiene velocidad igual a cero, se presenta un cambio brusco en el gradiente de velocidad. Este gradiente de velocidad en un sistema de flujo real, ocasiona que se creen fuerzas de corte cerca de los límites, reduciendo la velocidad del flujo cerca de ellas; a esto se le conoce como capa límite (Streeter y Wylie, 1980).

La Resistencia (R) tomada como potencia, es la pérdida que ofrece el aire al ser contraria al movimiento, para encontrar este dato se basa en la formulación de la ecuación (4) (MMPA, 2015).

$$
\mathrm{R}=\frac{1}{2} \rho \mathrm{v}^{3} \mathrm{~A}
$$

Acorde con las velocidades que desarrolle un camión se debe considerar el fenómeno aerodinámico del efecto suelo, que transcurre cuando la diferencia de presiones entre la zona superior del camión y la zona inferior del mismo se acercan más hacia la superficie terrestre, generando alteraciones en el flujo de aire que pueden aprovecharse en diversos campos. En sistemas de movilidad y transporte terrestre en lo posible, se debe establecer una zona de mayor presión por encima del vehículo y baja presión con el fluido que circula por debajo. Este diferencial de presiones provoca una succión que mantiene al vehículo contra el suelo, mejorando el agarre, lo que incrementa la posibilidad de ser más estable en curvas a velocidades superiores (Cembranos, 2013).

La Turbulencia implica que las distintas capas de aire se desplazan de manera caótica y desordenada. La formación y creación de flujos turbulentos dependen de la inercia y velocidad del viento sobre el camión. De esta manera, el número Reynolds (Coeficiente adimensional) servirá como indicador del incremento o decremento de la Resistencia aerodinámica. Entre los factores que pueden afectar a este indicador Reynolds (Re) se encuentra la velocidad del aire, su viscosidad (influida por factores climatológicos), y de la longitud de la forma analizada (García, 2005).

Los fenómenos atmosféricos son variados en la zona andina y en la región costanera y estos pueden afectar también en el comportamiento aerodinámico en camiones, pero estos no se pueden definir de manera exacta al momento de evaluar su afectación (Cooper, 2007); también dependerá de los ángulos del viento y de la geometría a la que se esté afectando.

La mayor fuente de incertidumbre con CFD es el modelado de la turbulencia en las capas de corte que se desarrollan alrededor del vehículo. La simulación CFD para el di- 
seño y el desarrollo aerodinámico está basada en realizar simulaciones de flujo estable por medio de modelos matemáticos para representar el efecto de la turbulencia en el campo de flujo promedio que rodean al vehículo.

Comúnmente las simulaciones y efectos de número de Reynolds en CFD son diferentes a las de los camiones porque los modelos de turbulencia se basan generalmente en un número de flujo Reynolds alto, donde la turbulencia tiende a comportarse de una manera diferente. Estas condiciones también niegan las características del flujo laminar y las capas de cizallamiento de transición que pueden ser frecuentes en la cara frontal de muchos vehículos de carretera. En los vehículos pesados, los flujos alrededor de las caras frontales del tractor son generalmente bajas con flujos muy diferentes a las características de la turbulencia, o, posiblemente, la ausencia de turbulencia que puede ocurrir sobre una gran parte de la cara frontal (National Research Council Canada, 2012).

Existen técnicas para simular los movimientos inestables a gran escala del flujo alrededor de un vehículo (Large Eddy Simulation) LES, y flujo separado (Detached Eddy Simulation) DES, pero las soluciones y resultados de estas técnicas son significativamente mayores en cuestiones de tiempo a las técnicas computacionales (National Research Council Canada, 2012).

\section{MATERIALES Y MÉTODOS}

Para el desarrollo del análisis se debe realizar una cuantificación de varios parámetros, mediante los cuales se podrá obtener un criterio apropiado acerca de la reacción aerodinámica que tiene la carrocería cuando se la expone al viento tratando de emular las situaciones reales en las cuales este semirremolque operará.

Los parámetros a analizar con la simulación CFD son:

- Drag (Arrastre)

- Lift (Sustentación)

- Número de Reynolds

- Resistencia Aerodinámica

- Velocidad de Flujo

- Presión

- Capa Límite

- Estela

- Turbulencia

- La Potencia para vencer la resistencia del aire $(\mathrm{Pa})$

- La resistencia del aire $(\mathrm{R})$

Para dimensionar las velocidades máximas a considerar, se tomó en cuenta el marco legal vigente en Ecuador del año 2015, el cual define una velocidad máxima de $100 \mathrm{~km} / \mathrm{h}$ para vehículos pesados; este parámetro se tomará como límite superior (INAMHI, 2010). 
La humedad, velocidad media y temperatura del aire fueron tomadas mediante los máximos obtenidos de las tablas 1 y 2 del anuario meteorológico, en las ciudades de Riobamba (Zona Andina) y Guayaquil (Zona Costanera) atendiendo a los siguientes datos:

Tabla 1. Características promedio del aire en Riobamba

\begin{tabular}{ccc}
\hline Temperatura & Humedad Relativa $\%$ & Velocidad media del Viento \\
\hline $22.7^{\circ} \mathrm{C}$ & $76 \%$ & $19.3 \mathrm{~km} / \mathrm{h}$ \\
\hline \multicolumn{3}{c}{ Fuente: INAMHI, 2010}
\end{tabular}

Tabla 2. Características promedio del aire en Guayaquil

\begin{tabular}{ccc}
\hline Temperatura & Humedad Relativa $\%$ & Velocidad media del Viento \\
\hline $32.6{ }^{\circ} \mathrm{C}$ & $80 \%$ & $40.5 \mathrm{~km} / \mathrm{h}$ \\
\hline \multicolumn{3}{c}{ Fuente: INAMHI, 2010 }
\end{tabular}

Es importante tener en cuenta los siguientes datos que serán constantes en el desarrollo de los diferentes estudios a realizar:

- Presión Atmosférica Riobamba: 0,704 atm.

- Presión Atmosférica Guayaquil: 0,998 atm.

- Densidad del Aire

Para determinar la densidad del aire en las diferentes ciudades en las que se realiza el estudio se la calcula mediante la ley de los gases ideales:

$$
\mathrm{PV}=\mathrm{nRT}
$$

Con la fórmula expuesta anteriormente (5) se obtiene las densidades para los microclimas seleccionados.

Tabla 3. Densidad del Aire

\begin{tabular}{cc}
\hline Zona - Región & Densidad $\left(\mathrm{Kg} / \mathrm{m}^{3}\right)$ \\
\hline Riobamba & 0.84 \\
Guayaquil & 1.15 \\
\hline
\end{tabular}

Para la simulación se debe establecer el tipo de material como influyente en el flujo, en este caso $0.04 \mathrm{~mm}$ (acero laminado nuevo), como rugosidad del material (White, 2013). Para el levantamiento de medidas se tomó como referencia un Semirremolque Cisterna para el Transporte de Cemento de fabricación nacional, tomando en cuenta las características estándar, sin adicionales. De esta manera se procede a construir el modelo 3D del transportador de cemento tipo cisterna (figura 1). 


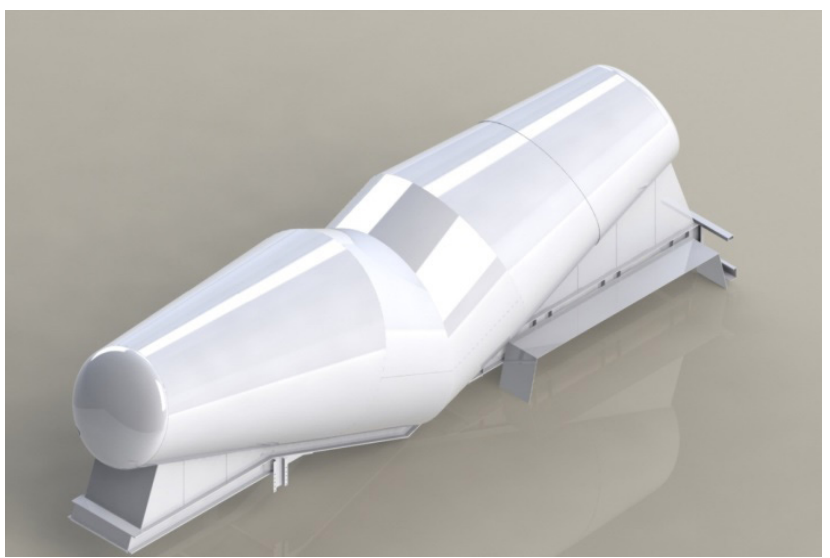

Figura 1. Modelo 3D Semirremolque Cisterna

Con la ayuda de un scanner 3D se procedió a obtener la nube de puntos de un tracto camión al azar (figura 2). Con la nube de puntos obtenida se procedió a unir los puntos más sobresalientes para con esto tener la sombra que definirá las líneas de flujo entrantes al semirremolque.
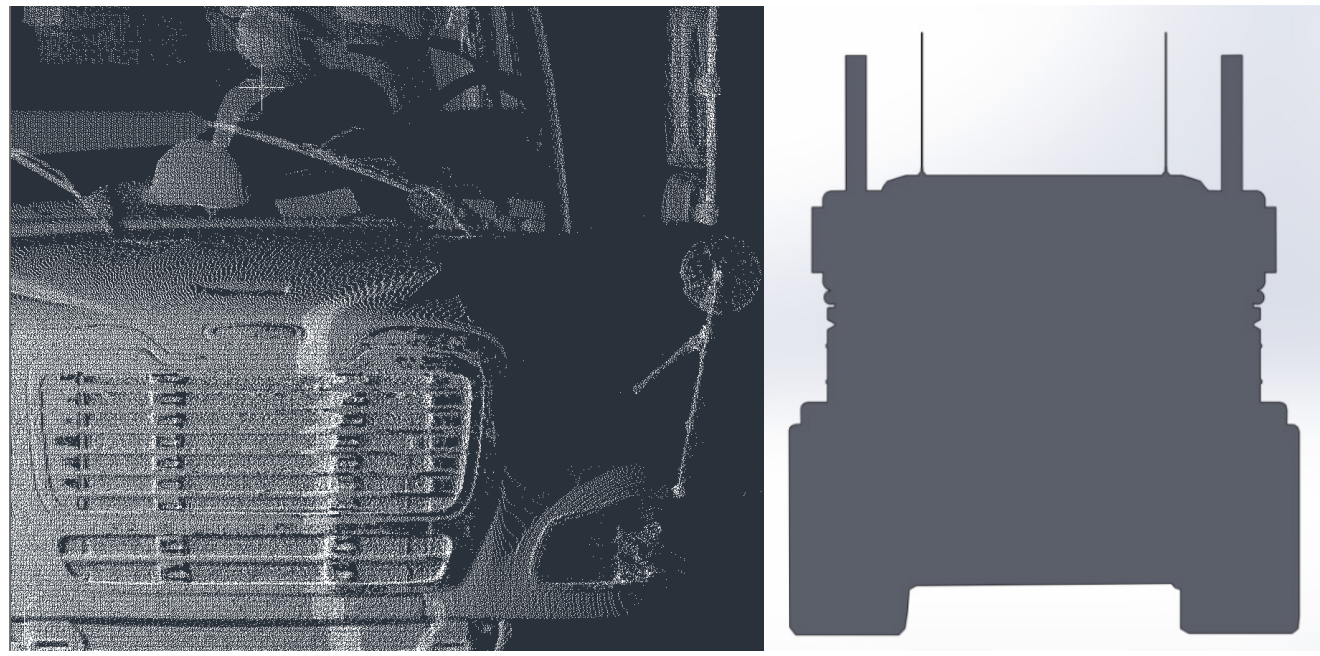

Figura 2. Obtención de la nube de puntos y simetría del tracto camión

Se establece las dimensiones en las cuales se crearán las condiciones de borde, limitando el análisis del modelo en un cubo de $18 \mathrm{~m}$ x $6 \mathrm{~m}$ x $4 \mathrm{~m}$, quedando el modelo ubicado en simetría con todas las paredes. Se realizó un análisis de la superficie externa del modelo, incluyendo espacios y cavidades donde circulará el fluido; dada la ubicación del modelo se tomará el eje «X» como referencia para el flujo del fluido.

Luego de realizar el trazado del modelo a analizar, planteadas las ecuaciones que lo gobiernan, establecidas las condiciones de contorno y configurados los resultados que se desea obtener se procede a la generación de una malla (figura 3), y posterior simulación. 
Se establece dos configuraciones de la simulación, con los datos ambientales tanto de la Sierra - Centro, Riobamba, como de la costa, Guayaquil y se procede a realizar la comparación de los datos obtenidos.

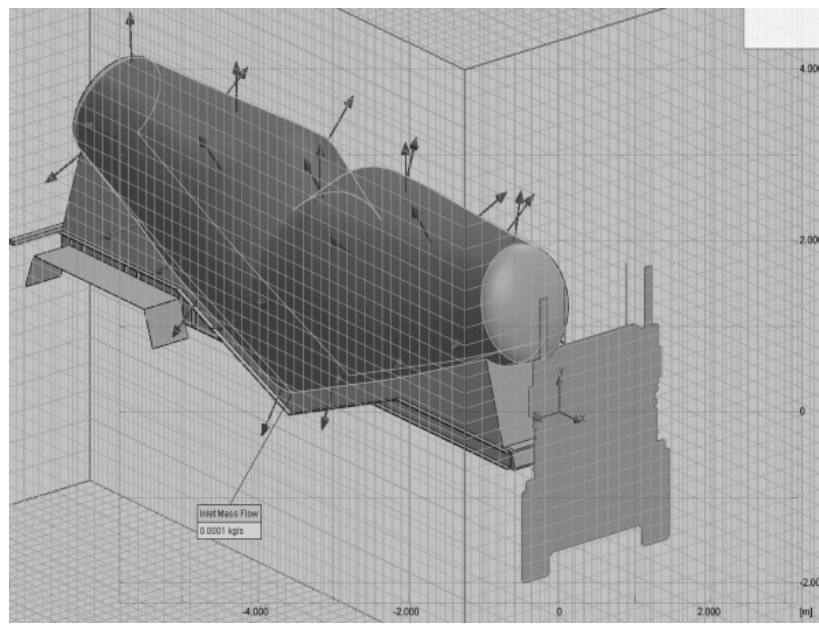

Figura 3. Mallado para posterior simulación

Dentro de las configuraciones, interiormente, en el software es necesario establecer datos exactos de los parámetros: tipo de fluido (laminar-turbulento), rugosidad, presión, temperatura, porcentaje de humedad (acorde con los microclimas), velocidad (25 $\mathrm{km} / \mathrm{h}-100 \mathrm{~km} / \mathrm{h}$ ), ubicación de sensores de viento, etc.

\section{RESULTADOS Y DISCUSIÓN}

Respecto al Coeficiente de Arrastre - Drag, se obtuvo un valor del semirremolque de 1.18, en lo concerniente al análisis de Sierra Centro - Riobamba y un valor de 0.86 en lo que corresponde al análisis del clima en la Costa - Guayas. Dado que la resistencia aerodinámica aumenta con el cuadrado de la velocidad, lo deseable es obtener un coeficiente de arrastre bajo, lo cual se traduciría en una disminución de la potencia para el arrastre, lo que deriva en menor consumo de combustible. Para aumentar la eficiencia se recomienda disminuir la distancia entre el tracto camión y el semirremolque al máximo posible, además de mejorar los perfiles aerodinámicos del cabezal.

En el caso del coeficiente de Sustentación - Lift se obtuvo un valor de 0.03 en el análisis del clima de Sierra Centro - Riobamba, igual al que se consiguió en la simulación realizada con el clima Costa - Guayas, valores que indican que el vehículo no tiene una fuerza considerable de empuje hacia el piso, se puede establecer que el modelo actual podría presentar inestabilidad al momento de ser expuesto a altas velocidades, aunque considerando el peso de la estructura esta inestabilidad podría no notarse.

En lo concerniente al número de Reynolds se obtuvo un valor de 3.0562 × $10^{6}$ para el análisis Sierra Centro - Riobamba, el cual indica que el fluido está en un régimen Turbulento, mientras que para el análisis con las características climáticas Costa-Guayas se 
obtuvo un valor de $4.1842 \times 10^{6}$, el cual muestra que al igual que en el estudio con el clima de la Sierra Centro se encuentra en régimen Turbulento.

Existen zonas en las que el fluido es laminar, gracias al diseño cónico del semirremolque que provoca un flujo adecuado el aire, y zonas donde se produce gran turbulencia, que es el caso del espacio que se genera entre el punto utilizado para el enganche con el tracto camión. Otro de los puntos donde se genera mayor turbulencia se encuentra en toda la « $\mathrm{V}$ » de descarga, en la parte frontal y posterior, y en todo el faldón inferior del semirremolque, puntos en los cuales se debe tomar en cuenta un suavizado de las superficies o una modificación en la estructura para que los componentes que generen turbulencia ingresen en el cono de transporte o espacios vacíos en la parte frontal o inferior del modelo, además de añadir paneles a los costados que permitan una mayor fluidez del aire. En las figuras 4 y 5 se puede observar las zonas en donde se genera mayor turbulencia, siendo estas las que se debe tener en cuenta para futuras modificaciones que mejoren el desempeño del semirremolque.

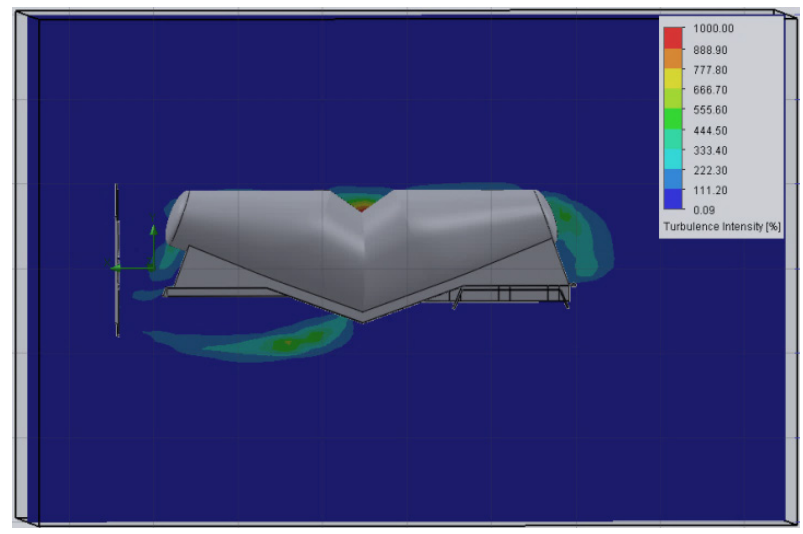

Figura 4. Intensidad de Turbulencia - Sierra - Centro

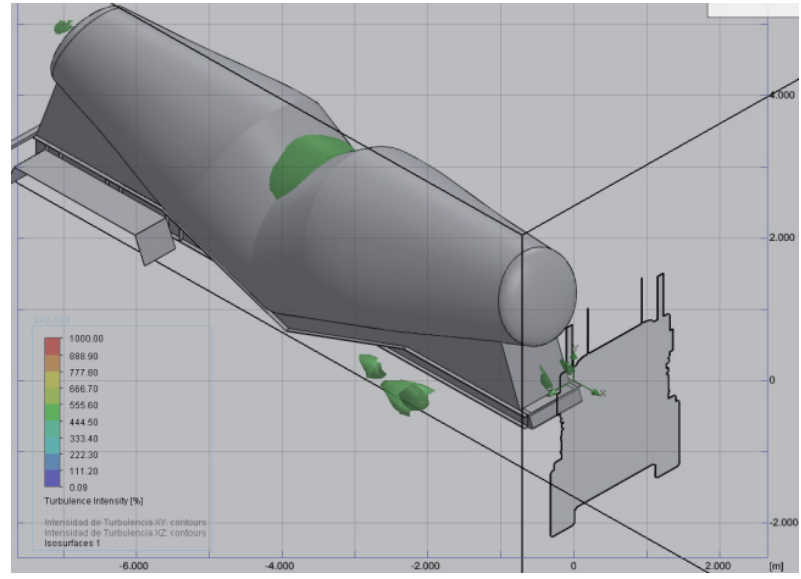

Figura 5. Puntos de Turbulencia Máxima - Sierra Centro

Para el caso de Resistencia Aerodinámica con las condiciones climáticas de la Sierra Centro, es decir con una densidad del aire calculada de $0.84 \frac{\mathrm{kg}}{\mathrm{m}^{3}}$, se obtuvo un valor 
máximo de 1706,69 (N), mientras que con el análisis en condiciones climáticas de la Costa, con una densidad del aire de $1.15 \frac{\mathrm{kg}}{\mathrm{m}^{3}}$, se obtuvo un valor máximo de 2336.55 (N). Ambos análisis se realizaron con variaciones de velocidad de $0 \mathrm{~km} / \mathrm{h}$ a $100 \mathrm{~km} / \mathrm{h}$ dando las curvas características:

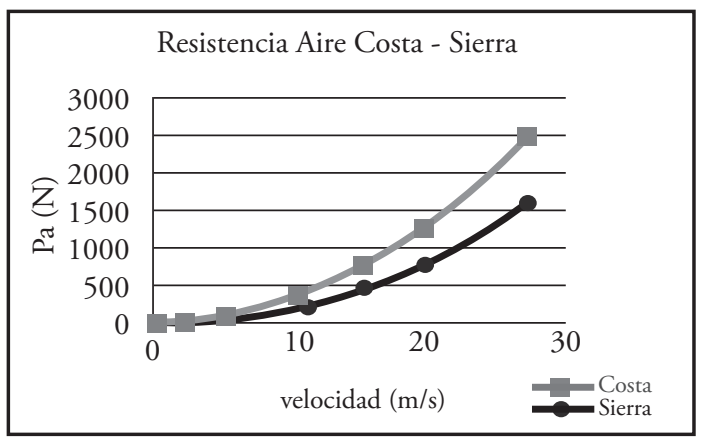

Figura 6. Resistencia del aire sector Costa y Sierra

La velocidad del flujo varía acorde con la forma del modelo, provocando perfiles de velocidad que influyen sobre el número Reynolds, lift y drag; es por esto que en ciertas zonas del modelo se encuentran Reynolds turbulentos, y fuerzas tanto de lift como drag considerables. Es importante tomar en cuenta los puntos donde los perfiles de velocidad son altos para suavizarlos y con esto reducir las turbulencias y las fuerzas que aumentan la potencia requerida para mover el vehículo.

Se puede establecer que los puntos a suavizar o corregir aerodinámicamente se encuentran en la zona de ingreso del flujo de aire, es decir en la cabina del tracto camión, de ahí que una solución óptima se basa en la utilización de alerones tanto en la parte superior como inferior del vehículo.

En cuanto al semirremolque los puntos críticos que se debe tomar en cuenta se encuentran en la parte inferior del mismo, dado que este punto es la unión de los dos cuerpos que forman la «V» de descarga; luego de este punto se puede observar una velocidad máxima de $4,91 \mathrm{~m} / \mathrm{s}$ como muestra la figura 7 .

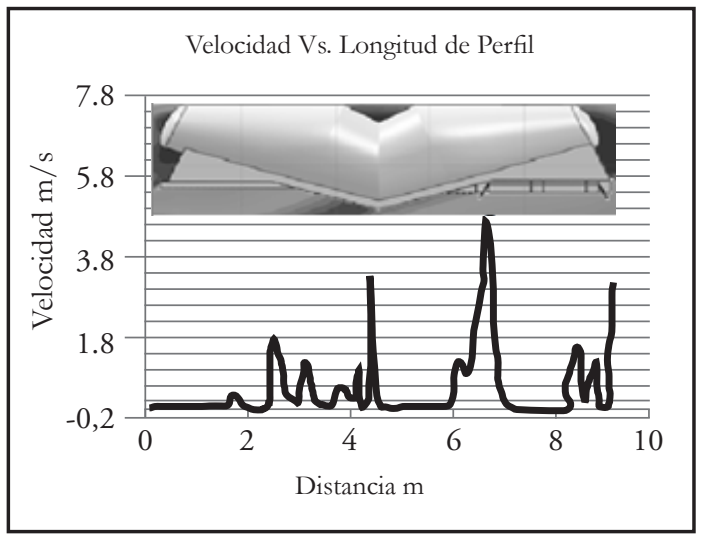

Figura 7. Velocidad del fluido respecto al perfil del semirremolque 
Es importante destacar que los resultados obtenidos en la simulación en las dos condiciones climáticas de Costa Guayas, tienen valores similares a los de la simulación Sierra Centro - Riobamba, por lo que las recomendaciones que se dan benefician a las dos configuraciones climáticas.

Al determinar el área de sección transversal frontal del modelo, como se aprecia en la figura 8, se puede calcular la Resistencia (Potencia) que consume el fluido a través de la ecuación (4), el área calculada por el software estima un valor de $6,615 \mathrm{~m}^{2}$. La resistencia que ofrece el aire al movimiento realizado con el clima de Riobamba da un valor de $\mathrm{R}=328,86 \mathrm{~W}$; en el caso del estudio con el clima de Guayaquil se obtuvo una resistencia $\mathrm{R}=391,511 \mathrm{~W}$.

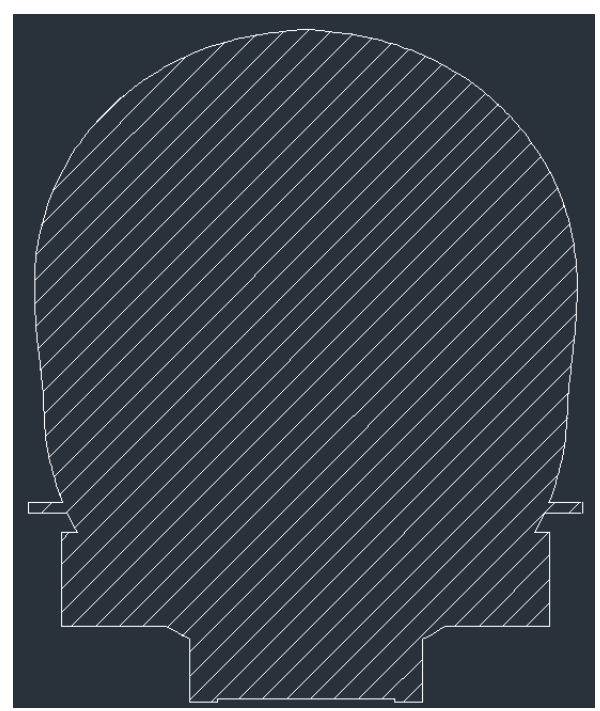

Figura 8. Sección Transversal Frontal

La presión estática que se genera en la carrocería por la diferencia de velocidades, puede crear cargas e influir en el comportamiento de la estructura cuando el semirremolque esté con carga y trabajando, por lo que se debe tomar en cuenta en el diseño estructural del modelo aquellos elementos que puedan deformarse o debilitarse por las mencionadas cargas.

Se puede establecer que la presión máxima del sistema en funcionamiento se encuentra en la pared superior - posterior de la « $V 》$ de descarga, la presión de acuerdo a la figura 9 es de 101168,9547 Pa en la Costa, y 71366,85061 Pa en la Sierra, similar a la presión que se genera en la parte inferior - posterior donde va instalado el eje que conecta los neumáticos. 


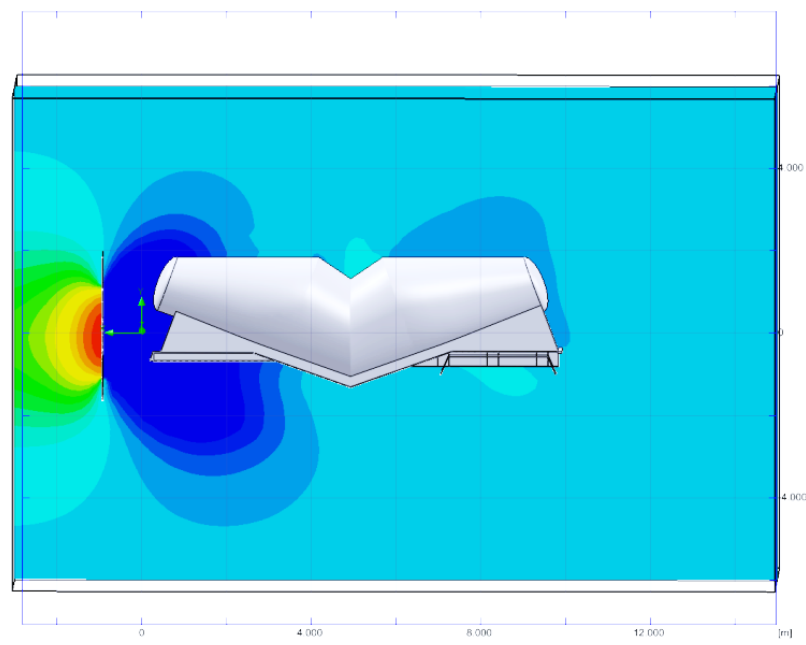

Figura 9. Presión respecto al perfil del semirremolque

El estudio de la capa límite permite observar la transición de velocidades en la franja de contacto entre el fluido y el camión semirremolque desplazándose. La formación de la capa límite dada entre otros factores por la viscosidad, es la causante de que el obstáculo produzca una variación en el movimiento de las líneas de corriente más próximas a él. El análisis de la estela tiene una importancia relevante, debido a que el comportamiento de esta, influye en la potencia que el vehículo tiene que ejercer para vencer la resistencia que ofrece el semirremolque, y debido a que esta fuerza no está en función de la sustentación se la considera como resistencia parásita, la forma de la estela varía por todas las pequeñas partes no aerodinámicas del vehículo (figura 10).

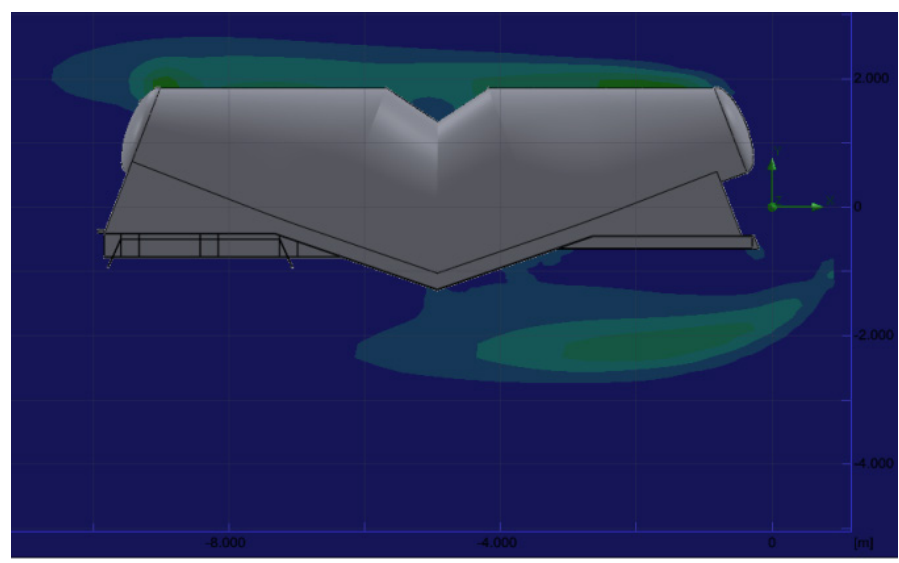

Figura 10. Estela del semirremolque

En el caso del semirremolque la forma redondeada del mismo no produce cambios importantes en la estela, lo cual implica que no se genera resistencias parásitas considerables. 


\section{CONCLUSIONES}

La potencia utilizada para vencer la resistencia al aire es superior en la zona costera, por lo que influye directamente la concentración de aire y la presión atmosférica; estos factores propios de los microclimas, a la par, también pueden influir positivamente en la combustión y por ende en la adición de potencia, viéndose compensada de alguna manera esta correspondencia.

El coeficiente de arrastre $\left(\mathrm{C}_{\mathrm{D}}\right)$ es inferior en la zona costera en comparación con la zona andina, a pesar de tener el mismo arrastre de forma y fricción, la densidad ayuda a mejorar notablemente este coeficiente, relacionado este fenómeno con la viscosidad del fluido causado por el diferencial de altura.

De forma general, el diseño del semirremolque es aerodinámicamente ideal por su terminación en cono y sus formas, produciendo el efecto que el aire tenga un flujo laminar a la salida, evitando con esto resistencias parásitas grandes que se provocan en las formas cuadradas; se debe evaluar la forma del casquete toriesférico para que el flujo del aire se optimice de mejor manera.

Se observó que es necesario obtener un coeficiente de resistencia aerodinámica $(\mathrm{Cx})$ adecuado y exacto, por lo que se hace imperioso realizar pruebas de campo con el tracto camión junto con el semirremolque con parámetros y condiciones monitoreadas, o la creación de un prototipo 3D que pueda ser monitoreado en un túnel de viento, para determinar el valor exacto e introducirlo en las formulaciones.

\section{REFERENCIAS BIBLIOGRÁFICAS}

Cembranos, Nistal y Florencio, Jesús. 2013. Planificación de la prueba y ajustes de los equipos y elementos de los sistemas de automatización industrial. España: Ediciones Paraninfo.

Coanda, Henri Marie. 2004. Aeronautics Learning Laboratory for Science Technology and Research. <http://www.allstar.fiu.edu/aero/Teachers.html> [Consulta: 17-102015].

Cooper, Watkins. 2007. The Unsteady Wind Environment of Road Vehicles - Part Two: Effects on Vebicle Development and Simulation of Turbulence, SAE.

García, Calixto. 2005. Zona Gravedad. <http://www.zonagravedad.com/- modules. php? name $=$ News $\&$ file $=$ article $\&$ sid $=776>$ [Consulta: $18-01-2015]$.

INAMHI, 2012. Anuario Meteorológico 2010. Informe Técnico. Quito: Instituto Nacional de Metereología e Hidrología.

Mc. Beath, Simon. 2005. Aerodinámica del Automóvil de Competición. Barcelona: CEAC.

MMPA - Minnesota Municipal Power Agency. 2015. Minnesota Municipal Power Agency. <http://www.mmpa.org/Uploaded_Files/ab/ab5c7c5c-79d9-48bd-b64d833001b7e230.pdf> [Consulta: 22-06-2015].

Mott, Robert. 2006. Mecánica de Fluidos. México: Pearson Educación. 
National Research Council Canada. 2012. «Review of Aerodynamic Drag Reduction - Devices for Heavy Trucks and Buses». Otawa: Centre for Surface Transportation Technology, Technical Report CST'T-HVC-TR-205.

SENPLADES. 2013. Plan Nacional para el Buen Vivir 2013-2017. 1 ra. ed. Quito.

Streeter, Victor y Wylie, Benjamin. 1980. Mecánica de los Fluidos. México: McGraw-Hill.

White, Frank. 2013. Mecánica de Fluidos. 5ta. ed. Madrid: McGraw-Hill.

Yung, Jo. 2001. Theory of Ground Vehicles. 3ra. ed. Otawa: Jhon Wiley.

Fecha de recepción: 12 de noviembre 2015

Fecha de aceptación: 01 de diciembre 2015

\section{Correspondencia}

Abel Remache Coyago

apremache@uce.edu.ec 